\title{
Análise Fatorial Confirmatória das Três Versões da Perceived Stress Scale (PSS): Um Estudo Populacional
}

\author{
Confirmatory Factor Analysis of Three Versions of the Perceived Stress Scale \\ (PSS): A Population-Based Study
}

\author{
André Faro* \\ Universidade Federal de Sergipe, Aracaju, SE, Brasil
}

\begin{abstract}
Resumo
Este estudo teve como objetivos realizar a análise fatorial confirmatória da Escala de Estresse Percebido (Perceived Stress Scale-PSS), nas versões traduzidas de 14, 10 e 4 itens; analisar sua validade concorrente com o Questionário de Saúde Geral (QSG-12); mapear o perfil do estresse em uma amostra representativa da população; e estabelecer parâmetros de avaliação do estresse por meio da normatização dos escores da população total. A amostragem foi de base populacional, em formato de conglomerado por setores censitários. Participaram 1.154 habitantes de uma capital do Nordeste brasileiro, sendo a maioria do sexo feminino $(55,6 \%)$, com nível de escolaridade em ensino médio $(51,7 \%)$, empregados $(72,7 \%)$ e não tabagistas $(90,6 \%)$. As medianas de renda e idade ficaram em R\$ 1.200,00 e 29 anos. Ao final, constatou-se que houve ajustes satisfatórios para as três versões da PSS apenas em seu modelo bifatorial e a escala com 10 itens se mostrou uma solução harmônica entre as versões completa e mais reduzida, considerando-se a relação entre a parcimônia na quantidade de itens e a robustez estatística deste instrumento. Ademais, a PSS ratificou sua validade concorrente com o QSG-12 e foi feita a normatização dos escores da PSS, estabelecendo-se parâmetros para comparação em futuros estudos.

Palavras-chave: Análise fatorial confirmatória, estresse, Escala de Estresse Percebido (PSS), Psicometria, validação de escalas.
\end{abstract}

\begin{abstract}
This study aimed to perform a confirmatory factor analysis of the Perceived Stress Scale (PSS) in the Brazilian versions of 14, 10, 4 items; to analyze their concurrent validity with the General Health Questionnaire (GHQ-12); to map the profile of the stress in a representative sampling; and to establish stress assessment parameters by the standardization of the scores from overall population. The sample was population-based by conglomerate designs and clusters tracts. Thus, 1,154 inhabitants from a capital city of a northeastern state of Brazil participated in the study, mostly female (55.6\%), with high school degree (51.7\%), employed (72.7\%), and nonsmokers $(90.6 \%)$. The average income and age were BRL 1,200.00 and 29 years old, respectively. By the end, it was found satisfactory adjustments for the three PSS versions but just in its two-factor model; the PSS with 10 items was shown as a harmonious solution between the full and reduced scales considering the relationship between parsimony and statistical robustness of the scale. Furthermore, the PSS ratified its concurrent validity with the GHQ-12. It was also made the scores normalization of the PSS setting up parameters for future comparisons.

Keywords: Confirmatory factor analysis, stress, Perceived Stress Scale (PSS), psychometry, scale validation
\end{abstract}

O estresse pode ser definido como um conjunto de processos de regulação do bem-estar, ativados frente a estímulos avaliados como produtores de mal-estar físico, social ou psicológico, e cujas repercussões são observadas por meio de alterações nos funcionamentos neuroendocrinológico e mental quando o indivíduo percebe estímulos

* Endereço para correspondência: Departamento de Psicologia, Centro de Educação de Ciências Humanas, Universidade Federal de Sergipe, Avenida Marechal Rondon, s/n, Jardim Rosa Elze, São Cristóvão, SE, Brasil 49100-000. E-mail: andre.faro.ufs@gmail.com como estressores (Goldstein \& Kopin, 2007; Lazarus \& Folkman, 1984; Monroe, 2008). Em virtude das transformações provocadas na dinâmica adaptativa física e psicológica, o estresse tem sido recorrentemente associado ao aumento na suscetibilidade ou facilitação para o desencadeamento de doenças, compondo modelos explicativos de variadas enfermidades (Cohen, Janick-Deverts, \& Miller, 2007; Holsboer \& Ising, 2010; Kajantie, 2008; McEwen, 2008).

Em bases de dados científicas se constata que o volume de pesquisas sobre o estresse e a saúde tem crescido 
marcantemente nas décadas recentes. Em um rápido levantamento de trabalhos que mencionem o estresse em seus resumos na plataforma Web of Science, em Agosto de 2013, com as palavras-chave "psychosocial stress or psychological stress", relacionadas ao termo "health", verifica-se o significativo incremento de publicações nos últimos 30 anos. Entre 1983 e 1993, 1.099 trabalhos utilizaram essas palavras-chave nos seus resumos. De 1993 a 2003, os descritores estão inseridos em cerca de 7.500 estudos e, nos últimos dez anos, de 2003 até 2013, quase 15.500 textos científicos incluíram esses termos.

Em vista de sua relevância no estudo da saúde e suas áreas de interface, instrumentos de pesquisa válidos e confiáveis são necessários na pesquisa do estresse. Assim, no âmbito das ciências psicológicas, Cohen, Kamarck e Mermelstein (1983) desenvolveram uma medida do estresse, a Escala de Estresse Percebido (Perceived Stress Scale [PSS]), possivelmente a escala mais utilizada para a mensuração cognitiva do estresse até hoje (Cohen \& Janick-Deverts, 2012; Lee, 2012). A PSS é originalmente composta por 14 itens (entre estudiosos, conhecida como PSS-14), mas também existem as versões reduzidas com 10 e 4 itens (PSS-10 e PSS-4), todas sendo utilizadas quando o objetivo é detectar em que grau os indivíduos avaliam situações e/ou estímulos de seu contexto experiencial como estressores (Cohen et al., 1983).

Quanto a sua validade, a PSS foi submetida a Análises Fatoriais Confirmatórias (AFC) em diferentes países, tais como Espanha (PSS-14, Remor, 2006), Japão (PSS-14, Mimura \& Griffiths, 2008), México (PSS-14, Ramírez \& Hernández, 2007), Jordânia (PSS-14, Almadi, Cathers, Mansour, \& Chow, 2012), China (as três versões da PSS, Leung, Lam, \& Chan, 2010), Portugal (as três versões da PSS, Ribeiro \& Marques, 2009), dentre outros.

No Brasil, Luft, Sanches, Mazo e Andrade (2007) realizaram a primeira tradução e validação para o contexto brasileiro, porém, a análise foi a fatorial exploratória da versão com 14 itens com idosos. Já Reis, Hino e Añez (2010) efetuaram a AFC da PSS-10 com um grupo de professores universitários, alcançando solução satisfatória, e Faro (2013) pesquisou o estresse em estudantes de pós-graduação, corroborando a estrutura fatorial encontrada por Luft et al. (2007). No entanto, não há, até então, um estudo que tenha conduzido a AFC da PSS no contexto brasileiro em uma amostra populacional e que, também, tenha testado simultaneamente as três versões. Isso permitiria o estabelecimento de um parâmetro geral para a avaliação do estresse nas escalas com 14, 10 e 4 itens, além do que favoreceria à normatização dos escores para comparações proporcionais entre diferentes amostras.

Enfim, considerando a relevância do instrumento nas pesquisas sobre o estresse e as lacunas ora apontadas, esta pesquisa objetivou: (a) Realizar a análise fatorial confirmatória da Escala de Estresse Percebido (PSS) nas versões traduzidas de 14, 10 e 4 itens; (b) Analisar sua validade concorrente com o Questionário de Saúde Geral
(QSG-12); (c) Mapear o perfil do estresse em uma amostra representativa da população; e (d) Estabelecer parâmetros de avaliação do estresse por meio da normatização dos escores da população total.

\section{Método}

\section{Participantes}

$\mathrm{O}$ delineamento da pesquisa foi transversal e de base populacional, através da técnica de amostragem por conglomerado, a partir de setores censitários delimitados no mapa oficial da cidade de Aracaju (SE). Para tanto, o cálculo amostral para distribuições que sigam a premissa da representatividade estatística indicou que seriam necessários 383 casos, considerando margem de erro em 5\% e nível de confiança em $95 \%$. Somado a esse montante, acrescentou-se $100 \%$ para compensar o efeito do delineamento ou eventuais perdas e casos extremos. Sendo assim, a amostra mínima esperada foi de 766 indivíduos.

Ao se estabelecer a divisão do município de Aracaju (SE) em cinco regiões geográficas (Norte, Sul, Centro, Leste e Oeste), sortearam-se aleatoriamente três bairros por região e, em cada bairro, dois quarteirões centrais para início da coleta de dados. Além desse controle, procedeu-se a classificação dos bairros conforme o status socioeconômico prevalente (atestada por juízes independentes), obtendo-se os estratos baixo, médio e alto. Com isso, sorteou-se um bairro por estrato em cada região.

A partir da primeira entrevista realizada, o que ocorria em uma das esquinas do quarteirão sorteado, saltava-se uma casa (à esquerda ou à direita) e convidava-se um morador da residência para participar da pesquisa, desde que possuísse idades entre 18 e 70 anos. Caso não houvesse ninguém apto a participar ou mesmo se a residência estivesse fechada, a visita era feita na casa seguinte. Foram previstos, ainda, os controles por sexo e turno de coleta, buscando-se a representatividade aproximada de 50/50 por sexo e os turnos para visita às casas foram proporcionalmente alternados (manhã, tarde e noite). Vale ressaltar que trabalhadores das residências (e.g., domésticas), visitantes (e.g., parentes), portadores de transtorno mental ou físico incapacitantes não fizeram parte da amostra, e apenas um morador por residência foi convidado a participar.

A partir dos critérios citados, a previsão mínima da coleta foi de 52 indivíduos por bairro, o que se realizou com o auxílio de uma equipe de 12 entrevistadores, devidamente identificados, treinados e supervisionados. Este estudo foi aprovado pelo Comitê de Ética em Pesquisa com Seres Humanos da Universidade Federal de Sergipe (UFS; CAAE - 0165.0.107.000-11).

\section{Instrumentos}

Para o mapeamento do perfil populacional, aplicou-se um questionário sociodemográfico contendo as variáveis sexo (masculino e feminino), idade (em anos), nível de escolaridade (até o fundamental, médio e superior), renda 
Faro, A. (2015). Análise Fatorial Confirmatória das Três Versões da Perceived Stress Scale (PSS): Um Estudo Populacional.

média individual nos últimos 3 meses (em reais) e ocupação (desempregado ou apenas estudante, empregado ou profissional liberal/autônomo). Além dessas variáveis, verificou-se a prevalência do tabagismo (sim ou não), mediante o critério do uso sistemático de fumo ou tabaco (pelo menos um cigarro, charuto, cachimbo ou cigarrilha) na semana anterior à coleta de dados.

A versão da Escala de Estresse Percebido (PSS) foi a traduzida e validada para o Brasil por Luft et al. (2007), que é composta por 14 itens sob a forma de perguntas, respondidos em escala do tipo Likert de 5 pontos ( 0 - Nunca a 4 -Sempre), com escore final entre 0 e 56 pontos. Os itens, seguindo a mesma proposta da versão original (Cohen et al., 1983), são divididos em sete negativos (1, 2, 3, 8, 11,12 e 14) e sete positivos $(4,5,6,7,9,10$ e 13$)$, sendo que este último fator tem sua pontuação invertida para o cômputo da pontuação geral. Mantendo-se a estrutura das versões abreviadas constantes na literatura (Cohen \& Janicki-Deverts, 2012; Leung et al., 2010), a versão com dez itens (PSS-10) teve seis itens no fator negativo $(1,2$, $3,8,11$ e 14) e quatro no positivo $(6,7,9$ e 10$)$, e a versão com quatro itens (PSS-4) ficou com dois negativos (2 e 14) e dois positivos (6 e 7).

Para a análise da validade convergente da PSS em suas três versões, aplicou-se o Questionário de Saúde Geral (QSG-12), que foi validado por Gouveia et al. (2003). O QSG-12 é uma breve e simples medida voltada ao diagnóstico geral (screen) dos transtornos mentais comuns, usualmente concebido como construto unifatorial (ver Gouveia, Lima, Gouveia, Freires, \& Barbosa, 2012). Os itens são apresentados como perguntas (e.g., Você tem notado que está agoniado? Você tem se sentido razoavelmente feliz?), separados em seis negativos (2, 5, 6, 9, 10 e 11) e seis positivos $(1,3,4,7,8$ e 12) e as respostas são assinaladas em escala de quatro pontos $(1-$ Absolutamente não a 4 - Muito mais que de costume). Para o cálculo final do índice de saúde geral, os itens negativos são submetidos ao procedimento recode. Geralmente o QSG-12 tem demonstrado índices satisfatórios de confiabilidade em diversas populações (Gouveia et al., 2012).

\section{Análises dos Dados}

Inicialmente, conduziram-se análises exploratórias e de ajuste no banco de dados para verificação das distribuições das variáveis segundo o modelo linear geral (normalidade, linearidade, homocedasticidade e singularidade), conforme recomendações de Tabachnick e Fidell (2007). Foram excluídos, da amostra total, 42 casos por situarem-se como valores extremos e outros 17 considerados como perdas por causa de preenchimento inadequado.

A partir dos escores finais das escalas e cálculo das demais variáveis cotejadas, foram obtidas as estatísticas descritivas (frequências absoluta e percentual, médias, desvios-padrão e medianas). Para as variáveis idade e renda média individual, inicialmente cotejadas de modo ordinal, mas que exibiram distribuições que não atendiam aos parâmetros de normalidade, efetuou-se a categorização em três níveis (faixa etária e faixa de renda), conforme distribuição percentílica de Tukey (Tukey's Hinge). Para a análise da confiabilidade das escalas e fatores executou-se o alfa de Cronbach, $(\alpha)$ aceitável acima de 0,60.

Nas Análises Fatoriais Confirmatórias (AFC) o método de estimação utilizado foi o da Máxima Verossimilhança (Maximum Likelihood [ML]). Para a avaliação do ajuste satisfatório dos modelos foram utilizados três índices: o GFI (Goodness of Fit Index; > 0,90), o CFI (Comparative Fit Index; > 0,90) e o RMSEA (Root Mean Square Error of Approximation; $\leq 0,08$; Intervalo de confiança entre 0 e 0,08$)$. A fim de efetuar a comparação entre os modelos, foram utilizados os seguintes índices: CAIC (Consistent Akaike Information Criterion) e o ECVI (Expected Cross-Validation Index). Para estes dois últimos índices, o modelo de menor valor é considerado o de melhor ajuste (Byrne, 2010; Garson, 2012).

A fim de realizar a normatização da pontuação da PSS, optou-se pela classificação geral em percentis (intervalos de 5\%) com a fórmula: "Escore $\mathrm{T}=($ EscoreZ da pontuação * 10) + 50". A partir da normatização segundo a população total, procedeu-se também a apresentação normatizada por sexo em cada uma das versões da PSS.

O nível de significância adotado foi de $p<0,05$ e para a avaliação da relação entre as variáveis foram aplicados os testes do Qui-Quadrado, $t$ de student, ANOVA (post-hoc LSD) e correlação de Pearson. Por fim, as análises inferenciais foram conduzidas através do programa Statistical Package for the Social Sciences (SPSS, v. 19), e as AFC's com o auxílio do programa Analysis of Moment Structures (AMOS, v. 18.0).

\section{Resultados}

\section{Análises Fatoriais Confirmatórias e Análises de Confiabilidade das Três Versões da PSS}

A análise dos indicadores de ajustamento exibidos na Tabela 1 demonstra que apenas o modelo bifatorial apresenta ajustes satisfatórios em todos os índices cotejados, exceto no caso da PSS-4, que ambas as condições denotam satisfatório resultado, mas, ainda assim, o unifatorial aponta para uma melhor solução. Vale ainda notar que o parâmetro de comparação de modelos (CAIC) e o de expectativa de validação em amostras similares (ECVI) mostrou que o desenho bifatorial é superior em todas as versões.

Tendo em vista que os melhores ajustes foram obtidos com o modelo bifatorial da PSS, na Tabela 2 estão relacionados os pesos de regressão padronizados (cargas fatoriais) dos itens nas três versões. Nela se constata que todos os itens tiveram pesos acima de 0,40 , exceto o item 12 , no fator negativo da PSS -14, que foi de 0,154 . Isso significa que apenas $15,4 \%$ da variabilidade amostral do item foi explicada pelo fator que o agrega; sendo este, um possível critério para exclusão do item, caso se buscasse um modelo 
Psicologia: Reflexão e Crítica, 28(1), 21-30.

Tabela 1

Resultados da Análise Fatorial Confirmatória da PSS em suas Três Versões (14, 10 e 4 itens)

$\begin{array}{lllll}\mathrm{X}^{2}(g l) & \text { GFI } & \text { CFI } & \text { CAIC } & \text { ECVI (IC) }\end{array}$

PSS-14

$\begin{array}{rllllll}\text { Uni } & 792,889(77)^{*} & 0,889 & 0,785 & 1018,317 & 0,736(0,661-0,818) & 0,090(0,084-0,096) \\ \text { Bi } & 389,989(76)^{*} & 0,951 & 0,906 & 623,468 & 0,389(0,338-0,445) & 0,060(0,054-0,066)\end{array}$

PSS-10

$\begin{array}{rllllll}\text { Uni } & 442,331(36)^{*} & 0,919 & 0,827 & 595,299 & 0,417(0,361-0,479) & 0,099(0,091-0,107) \\ \text { Bi } & 191,720(34)^{*} & 0,967 & 0,933 & 360,790 & 0,203(0,168-0,244) & 0,063(0,055-0,072)\end{array}$

PSS-4

\begin{tabular}{rllllll} 
Uni & $26,109(3)^{*}$ & 0,988 & 0,958 & 82,466 & $0,035(0,024-0,052)$ & $0,082(0,055-0,112)$ \\
Bi & $<0,001(1)^{* *}$ & 1,000 & 1,000 & 72,459 & $0,016(0,016-0,016)$ & $0,000(0,000-0,000)$ \\
\hline
\end{tabular}

Notas. Uni = Modelo unifatorial; $\mathrm{Bi}=$ Modelo bifatorial. $\mathrm{X}^{2}(\mathrm{~g} l)=$ Qui-quadrado (graus de liberdade); GFI = Goodness of Fit Index; $\mathrm{CFI}=$ Comparative Fit Index; CAIC = Consistent Akaike Information Criterion; ECVI = Expected Cross-Validation Index; RMSEA $=$ Root Mean Square Error of Approximation.

antervalo de confiança.

${ }^{*} p<0,001 ;{ }^{* *} p=0,985$.

parcimonioso. No entanto, ao se analisar a confiabilidade, viu-se que o alfa do fator negativo da PSS-14 elevar-se-ia de 0,707 apenas para 0,730 , caso fosse excluído o item 12. Assim, optou-se por não excluir o item e manter a estrutura original, já que o ajuste foi satisfatório e não se alcançaria uma substancial elevação na confiabilidade. Esse problema e solução também foi visto em outro estudo (Ramírez \& Hernández, 2007), o qual seguiu a mesma decisão aqui tomada.

Ainda na Tabela 2, vê-se que as correlações entre os fatores foram elevadas nas três versões, com destaque para a PSS-4, por ter sido o mais alto valor $(-0,771)$, embora possua os menores valores no alfa de Cronbach. No caso da PSS-10, faz-se notar que ela apresenta o maior índice de confiabilidade da escala total $(0,792)$ e exibiu cargas fatoriais desde 0,420 até 0,638 . Ao se correlacionar as escalas completas, todas as relações, conforme esperado, foram estatisticamente significativas em $p<0,001$, com os seguintes valores: PSS-14 e PSS-10 $=0,967$, PSS-14 e PSS-4 $=0,854$, PSS-10 e PSS-4 $=0,871$.

\section{Perfil Populacional e Distribuição do Estresse}

A amostra final contou com 1.154 indivíduos, alcançando margem de erro de aproximadamente $3 \%$ e nível de confiança em $95 \%$. Na caracterização dos participantes, $55,6 \%(n=642)$ foi do sexo feminino e $44,4 \%(n=512)$ do masculino, com mediana de idade na amostra geral em 29 anos e estratos etários distribuídos desta forma: $26,3 \%$ $(n=304)$ com até 23 anos, 50,6\% $(n=584)$ entre 23 e
41 anos e $23,1 \%(n=286)$ acima de 41 anos de idade. Em relação à escolaridade, predominou o ensino médio $(51,7 \% ; n=597)$ e, em seguida, superior $(30,6 \% ; n=$ $353)$ e fundamental $(17,7 \% ; n=204)$. Obteve-se $72,7 \%$ de ocupação no mercado de trabalho e $9,4 \%$ foi identificado como tabagista $(n=108)$. No quesito renda, a mediana de renda individual na amostra geral foi de $\mathrm{R} \$ 1.200,00$, com 26,8\% ( $n=309)$ ganhando até $\mathrm{R} \$ 600,00,51,0 \%(n$ $=588)$ entre $\mathrm{R} \$ 600,00$ e $\mathrm{R} \$ 2.000,00$ e $22,3 \%(n=257)$ acima de $\mathrm{R} \$ 2.000,00$.

No que se refere à pontuação média nas escalas (Tabela 3), percebe-se que a média da PSS-14 apontou para um ponto de corte de $50 \%$ do escore total ( $24 \mathrm{em} 48$ pontos), enquanto que a PSS-10 e a PSS-4 tiveram a pontuação média ligeiramente abaixo, em aproximadamente 47\% (17 em 36 pontos) e $37 \%$ (6 em 16 possíveis), respectivamente. Tais achados mostram que principalmente a PSS-14 denota boa capacidade discriminativa ao longo da curva normal do estresse, seguida de perto pela PSS-10, cuja média de pontos nos fatores foram praticamente iguais. No caso da PSS-4, a média indica tendência a subestimar a variabilidade total da escala, ou seja, as pontuações variaram acima da média da proporção esperada (50/50). 
Faro, A. (2015). Análise Fatorial Confirmatória das Três Versões da Perceived Stress Scale (PSS): Um Estudo Populacional.

Tabela 2

Pesos de Regressão Padronizados (Cargas Fatoriais), Índices de Correlação e Confiabilidade do Modelo Bifatorial das Três Versões da PSS

\begin{tabular}{|c|c|c|c|c|c|c|}
\hline \multirow[b]{2}{*}{ Neste último mês, com que frequência você tem... } & \multicolumn{2}{|c|}{ PSS-14 } & \multicolumn{2}{|c|}{ PSS-10 } & \multicolumn{2}{|c|}{ PSS-4 } \\
\hline & Neg & Pos & Neg & Pos & Neg & Pos \\
\hline 1. Ficado triste por causa de algo que aconteceu inesperadamente? & 0,532 & 0 & 0,531 & 0 & - & - \\
\hline 2. Se sentido incapaz de controlar coisas importantes em sua vida? & 0,597 & 0 & 0,593 & 0 & 0,614 & 0 \\
\hline 3. Se sentido nervoso e estressado? & 0,583 & 0 & 0,581 & 0 & - & - \\
\hline $\begin{array}{l}\text { 8. Achado que não conseguiria lidar com todas as coisas que você tem que } \\
\text { fazer? }\end{array}$ & 0,427 & 0 & 0,420 & 0 & - & - \\
\hline 11. Ficado irritado porque as coisas que acontecem estão fora do seu controle? & 0,636 & 0 & 0,638 & 0 & - & - \\
\hline 12. Se encontrado pensando sobre as coisas que deve fazer? & 0,154 & 0 & - & - & - & - \\
\hline $\begin{array}{l}\text { 14. Sentido que as dificuldades se acumulam a ponto de você acreditar que } \\
\text { não pode superá-las? }\end{array}$ & 0,592 & 0 & 0,596 & 0 & 0,608 & 0 \\
\hline 4. Tratado com sucesso dos problemas difíceis da vida? & 0 & 0,536 & - & - & - & - \\
\hline $\begin{array}{l}\text { 5. Se sentido que está lidando bem com as mudanças importantes que estão } \\
\text { ocorrendo em sua vida? }\end{array}$ & 0 & 0,628 & - & - & - & - \\
\hline 6. Se sentido confiante na sua habilidade de resolver problemas pessoais? & 0 & 0,674 & 0 & 0,595 & 0 & 0,637 \\
\hline 7. Sentido que as coisas estão acontecendo de acordo com a sua vontade? & 0 & 0,577 & 0 & 0,591 & 0 & 0,563 \\
\hline 9. Conseguido controlar as irritações em sua vida? & 0 & 0,560 & 0 & 0,582 & - & - \\
\hline 10. Sentido que as coisas estão sob seu controle? & 0 & 0,636 & 0 & 0,692 & - & - \\
\hline 13. Conseguido controlar a maneira como gasta seu tempo? & 0 & 0,418 & - & - & - & - \\
\hline Correlação entre fatores & \multicolumn{2}{|c|}{$-0,624$} & \multicolumn{2}{|c|}{$-0,664$} & \multicolumn{2}{|c|}{$-0,771$} \\
\hline \multirow{2}{*}{ Alfa de Cronbach } & 0,707 & 0,772 & 0,733 & 0,705 & 0,543 & 0,533 \\
\hline & \multicolumn{2}{|c|}{0,775} & \multicolumn{2}{|c|}{0,792} & \multicolumn{2}{|c|}{0,640} \\
\hline
\end{tabular}

Notas. Neg = Fator Negativo; Pos = Fator Positivo. Para o cálculo do alfa de Cronbach da escala total os valores dos itens positivos foram invertidos.

Tabela 3

Estatística Descritiva e Comparação por Sexo da Pontuação nas Escalas e nos Fatores da PSS-14, PSS-10 e PSS-4

\begin{tabular}{|c|c|c|c|c|c|c|c|}
\hline & \multicolumn{2}{|c|}{ População } & \multicolumn{2}{|c|}{ Masculino } & \multicolumn{2}{|c|}{ Feminino } & \multirow{2}{*}{$p$} \\
\hline & Mín-Max & $M(D P)$ & Mín-Max & $M(D P)$ & Mín-Max & $M(D P)$ & \\
\hline \multicolumn{8}{|l|}{ PSS-14 } \\
\hline Total & $2-48$ & $24,0(8,23)$ & $3-48$ & $22,9(8,20)$ & $2-47$ & $24,7(8,17)$ & $<0,00$ \\
\hline Neg & $0-27$ & $13,9(4,89)$ & $2-27$ & $13,2(4,72)$ & $0-27$ & $14,5(4,94)$ & $<0,00$ \\
\hline Pos & $2-28$ & $18,0(4,78)$ & $2-28$ & $18,2(4,73)$ & $4-28$ & $17,8(4,81)$ & 0,132 \\
\hline \multicolumn{8}{|l|}{ PSS-10 } \\
\hline Total & $0-36$ & $17,0(6,67)$ & $1-35$ & $15,8(6,59)$ & $0-36$ & $17,6(6,63)$ & $<0,00$ \\
\hline $\mathrm{Neg}$ & $0-24$ & $10,9(4,63)$ & $0-23$ & $10,1(4,45)$ & $0-24$ & $11,4(4,69)$ & $<0,00$ \\
\hline Pos & $0-16$ & $10,0(3,07)$ & $0-16$ & $10,2(3,04)$ & $1-16$ & $9,8(3,07)$ & 0,008 \\
\hline \multicolumn{8}{|l|}{ PSS-4 } \\
\hline Total & $0-16$ & $6,0(3,18)$ & $0-15$ & $5,7(3,10)$ & $0-16$ & $6,1(3,23)$ & 0,016 \\
\hline Neg & $0-8$ & $3,0(2,03)$ & $0-8$ & $2,8(1,93)$ & $0-8$ & $3,1(2,10)$ & 0,020 \\
\hline Pos & $0-8$ & $5,0(1,75)$ & $0-8$ & $5,1(1,73)$ & $0-8$ & $4,9(1,75)$ & 0,093 \\
\hline
\end{tabular}

Notas. Total = Escala completa; Neg = Fator Negativo; Pos = Fator Positivo; Min-Max = Mínimo-Máximo; $M(D P)=$ Média (Desvio-Padrão); $p=p$-valor com o teste $t$ de Student. 
Na comparação das pontuações por sexo, viu-se que, exceto no fator positivo da PSS-14 e da PSS-4, todas as demais condições analisadas mostraram significância estatística, com o seguinte desfecho: as mulheres apresentam maior pontuação média no estresse nas três versões e, quando decompostas por fatores, o mesmo ocorre no negativo.

$\mathrm{Na}$ Tabela 4 estão relacionadas as pontuações nas três versões da PSS segundo as demais variáveis do perfil populacional. Com efeito, no que se refere à faixa etária, só houve diferença na comparação entre indivíduos com mais de 41 anos e menos de 23 anos, sendo que estes últimos apresentam maior pontuação média. Não foram encontradas diferenças nas versões com 10 e 4 itens. Para a escolaridade, o nível superior apresentou menor média nas três versões e, quanto à faixa de renda, aqueles que ganhavam mais de R $\$ 2000$ também exibiram menor estresse em todas as versões. Tabagistas e os não empregados também exibiram pontuações mais altas de estresse quando comparados aos não tabagistas e empregados, exceto na PSS-4, que não houve diferença quanto à ocupação.

\section{Perfil do QSG na População e Validade Concorrente da PSS}

Para a análise da validade concorrente, foram executadas correlações de Pearson entre a PSS e o QSG. Com efeito, constatou-se que todas as versões da PSS, tanto a completa com em seus respectivos fatores, exibiram significância estatística quando correlacionados à pontuação na escala de saúde geral $(p<0,001)$. Para a PSS-14, a correlação da escala total foi de - 0,294 e os fatores negativo e positivo tiveram, ordenadamente, os seguintes valores: - 0,272 e 0,227. Na PSS-10, a escala total foi de - 0,296, o fator negativo $-0,279$ e o positivo 0,222 . Por fim, a PSS-4 total apresentou correlação em - 0,287 e com os fatores negativo e positivo em - 0,274 e 0,204, respectivamente. Na presente amostra, a média geral do QSG ficou em 35,6 pontos $(D P=6,02)$, com extremos em 16 e 48 pontos. Neste estudo, o alfa de Cronbach da QSG foi de 0,75.

Normatização dos Escores e Parametrização dos Índices Por fim, realizou-se a normatização dos índices da PSS14, PSS-10 e PSS-4, conforme a distribuição amostral total e por sexo (Tabela 5). O Escore T classifica a distribuição com média padronizada em 50 e desvio-padrão 10, o que permite a parametrização da pontuação bruta em uma escala que pode ser compreendida numa variação desde 0 até 100 , propiciando o estabelecimento de um prisma para comparação entre populações.

\section{Tabela 4}

Estatística Descritiva e Comparação do Perfil Populacional nas Escalas PSS-14, PSS-10 e PSS-4

\begin{tabular}{|c|c|c|c|}
\hline & PSS-14* & PSS-10* & PSS-4* \\
\hline \multicolumn{4}{|l|}{ Faixa etária ${ }^{1}$} \\
\hline Até 23 anos & $24,8(8,24)^{\mathrm{a}}$ & $17,1(6,66)^{\mathrm{c}}$ & $6,0(3,21)^{\mathrm{c}}$ \\
\hline Entre 23 e 41 anos & $23,8(8,20)^{\mathrm{a}, \mathrm{b}}$ & $16,8(6,65)^{\mathrm{c}}$ & $5,8(3,15)^{\mathrm{c}}$ \\
\hline Acima de 41 anos & $23,0(8,20)^{\mathrm{b}}$ & $16,6(6,74)^{\mathrm{c}}$ & $6,1(3,19)^{\mathrm{c}}$ \\
\hline \multicolumn{4}{|l|}{ Escolaridade $^{1}$} \\
\hline Até o fundamental & $25,0(8,22)^{\mathrm{a}}$ & $18,1(6,71)^{\mathrm{a}}$ & $6,4(3,41)^{\mathrm{a}}$ \\
\hline Médio & $24,4(8,18)^{\mathrm{a}}$ & $17,2(6,62)^{\mathrm{a}}$ & $6,1(3,16)^{\mathrm{a}}$ \\
\hline Superior & $22,3(8,11)^{b}$ & $15,5(6,52)^{b}$ & $5,4(3,00)^{\mathrm{b}}$ \\
\hline \multicolumn{4}{|l|}{ Faixa de Renda ${ }^{1}$} \\
\hline Até $\mathrm{R} \$ 600$ & $25,3(8,43)^{\mathrm{a}}$ & $17,8(6,72)^{\mathrm{a}}$ & $6,2(3,17)^{\mathrm{a}}$ \\
\hline Entre $\mathrm{R} \$ 600$ e $\mathrm{R} \$ 2000$ & $24,3(8,18)^{\mathrm{a}}$ & $17,1(6,71)^{\mathrm{a}}$ & $6,0(3,25)^{\mathrm{a}}$ \\
\hline Mais de $\mathrm{R} \$ 2000$ & $21,3(7,51)^{b}$ & $15,0(6,19)^{\mathrm{b}}$ & $5,3(2,93)^{b}$ \\
\hline \multicolumn{4}{|l|}{ Tabagismo $^{2}$} \\
\hline Sim & $26,3(8,62)^{a}$ & $18,9(6,83)^{\mathrm{a}}$ & $6,6(3,55)^{\mathrm{a}}$ \\
\hline Não & $23,6(8,15)^{b}$ & $16,6(6,62)^{\mathrm{b}}$ & $5,8(3,13)^{\mathrm{b}}$ \\
\hline \multicolumn{4}{|l|}{ Ocupação $^{2}$} \\
\hline Empregado & $23,7(8,45)^{\mathrm{a}}$ & $16,5(6,84)^{\mathrm{a}}$ & $5,9(3,25)^{\mathrm{c}}$ \\
\hline Desempregado & $24,8(7,67)^{\mathrm{b}}$ & $17,5(6,23)^{b}$ & $6,0(2,92)^{\mathrm{c}}$ \\
\hline
\end{tabular}

Notas. * Média (Desvio-Padrão). ${ }^{1}$ ANOVA (post-hoc LSD); ${ }^{2} t$ de Student; $p<0,05 ;{ }^{\mathrm{a}} \mathrm{e}^{\mathrm{b}}$ diferem em $p<0,05 ;{ }^{\mathrm{c}}$ não há diferença $(p>0,05)$. 
Faro, A. (2015). Análise Fatorial Confirmatória das Três Versões da Perceived Stress Scale (PSS): Um Estudo Populacional.

Tabela 5

Normatização dos Escores da PSS, segundo a Distribuição Total e por Sexo

\begin{tabular}{|c|c|c|c|c|c|c|c|c|c|c|c|c|c|c|c|c|c|c|}
\hline \multirow{3}{*}{ Percentil } & \multicolumn{6}{|c|}{ PSS-14 } & \multicolumn{6}{|c|}{ PSS-10 } & \multicolumn{6}{|c|}{ PSS-4 } \\
\hline & \multicolumn{3}{|c|}{ Pontuação } & \multicolumn{3}{|c|}{ Escore $\mathrm{T}$} & \multicolumn{3}{|c|}{ Pontuação } & \multicolumn{3}{|c|}{ Escore $\mathrm{T}$} & \multicolumn{3}{|c|}{ Pontuação } & \multicolumn{3}{|c|}{ Escore $\mathrm{T}$} \\
\hline & $\mathrm{T}$ & M & $\mathrm{F}$ & $\mathrm{T}$ & M & $\mathrm{F}$ & $\mathrm{T}$ & M & $\mathrm{F}$ & $\mathrm{T}$ & M & $\mathrm{F}$ & $\mathrm{T}$ & $\mathrm{M}$ & $\mathrm{F}$ & $\mathrm{T}$ & $\mathrm{M}$ & $\mathrm{F}$ \\
\hline 5 & 11 & 11 & 12 & 34 & 34 & 35 & 7 & 6 & 7 & 35 & 34 & 35 & 1 & 1 & 1 & 34 & 34 & 34 \\
\hline 10 & 14 & 13 & 14 & 38 & 37 & 38 & 9 & 8 & 9 & 37 & 37 & 38 & 2 & 2 & 2 & 38 & 38 & 38 \\
\hline 15 & 15 & 15 & 16 & 39 & 39 & 40 & 10 & 9 & 11 & 40 & 38 & 41 & 3 & 2 & 3 & 41 & 38 & 41 \\
\hline 20 & 17 & 16 & 17 & 42 & 40 & 42 & 11 & 10 & 12 & 41 & 40 & 43 & 3 & 3 & 3 & 41 & 41 & 41 \\
\hline 25 & 18 & 17 & 19 & 43 & 42 & 44 & 12 & 11 & 13 & 43 & 41 & 44 & 4 & 3 & 4 & 44 & 41 & 44 \\
\hline 30 & 19 & 18 & 20 & 44 & 43 & 45 & 13 & 12 & 14 & 44 & 43 & 46 & 4 & 4 & 4 & 44 & 44 & 44 \\
\hline 35 & 20 & 19 & 22 & 45 & 44 & 48 & 14 & 13 & 15 & 46 & 44 & 47 & 4 & 4 & 5 & 44 & 44 & 47 \\
\hline 40 & 22 & 20 & 23 & 48 & 45 & 49 & 15 & 13 & 16 & 47 & 44 & 49 & 5 & 5 & 5 & 47 & 47 & 47 \\
\hline 45 & 23 & 21 & 24 & 49 & 46 & 50 & 16 & 14 & 17 & 49 & 46 & 50 & 5 & 5 & 6 & 47 & 47 & 50 \\
\hline 50 & 24 & 22 & 25 & 50 & 48 & 51 & 16 & 15 & 18 & 49 & 47 & 52 & 6 & 5 & 6 & 50 & 47 & 50 \\
\hline 55 & 25 & 23 & 26 & 51 & 49 & 52 & 17 & 16 & 18 & 50 & 49 & 52 & 6 & 6 & 6 & 50 & 50 & 50 \\
\hline 60 & 26 & 24 & 27 & 52 & 50 & 54 & 18 & 17 & 19 & 52 & 50 & 53 & 7 & 6 & 7 & 53 & 50 & 53 \\
\hline 65 & 27 & 26 & 28 & 54 & 52 & 55 & 19 & 18 & 20 & 53 & 52 & 55 & 7 & 7 & 7 & 53 & 53 & 53 \\
\hline 70 & 28 & 27 & 29 & 55 & 54 & 56 & 20 & 19 & 21 & 55 & 53 & 56 & 7 & 7 & 8 & 53 & 53 & 56 \\
\hline 75 & 29 & 28 & 30 & 56 & 55 & 57 & 21 & 20 & 22 & 56 & 55 & 58 & 8 & 8 & 9 & 56 & 56 & 60 \\
\hline 80 & 31 & 29 & 32 & 59 & 56 & 60 & 23 & 21 & 24 & 59 & 57 & 61 & 9 & 8 & 9 & 60 & 56 & 60 \\
\hline 85 & 33 & 32 & 34 & 61 & 60 & 62 & 24 & 23 & 25 & 61 & 59 & 62 & 10 & 9 & 10 & 63 & 60 & 63 \\
\hline 90 & 35 & 34 & 36 & 63 & 62 & 65 & 26 & 25 & 27 & 64 & 62 & 65 & 10 & 10 & 11 & 63 & 63 & 66 \\
\hline 95 & 38 & 39 & 38 & 67 & 68 & 67 & 29 & 28 & 29 & 68 & 67 & 68 & 12 & 12 & 12 & 69 & 69 & 69 \\
\hline
\end{tabular}

Notas. $\mathrm{T}=$ População total; $\mathrm{M}=$ Sexo masculino; $\mathrm{F}=$ Sexo feminino.

Como visto na Tabela 5, os percentis 5 e 95 tiveram, na população total, estes escores $\mathrm{T}$, respectivamente: PSS-14 34 (11 pontos) e 67 (38 pontos); na PSS-10, 35 ( 7 pontos) e 68 ( 29 pontos) e na PSS-4, 34 (1 ponto) e 69 (12 pontos). Os escores T médios (percentil 50) foram os seguintes: na PSS-14, 24 pontos; na PSS-10, 16 pontos, e na PSS-4, 6 pontos.

\section{Discussão}

Esta pesquisa objetivou, principalmente, realizar a $\mathrm{AFC}$ da PSS nas versões de 14, 10 e 4 itens. Em vista disso, encontrou-se que as escalas, em modo bifatorial, exibem excelentes ajustes nas AFC's. Quanto à validade interna, especialmente no caso da PSS-4, é necessário pontuar que embora esteja abaixo do ponto de corte usualmente considerado adequado, o alfa sofre impacto quando o número de itens de uma escala/fator é pequeno, o que minimiza a variabilidade da distribuição, sem que isso necessariamente comprometa a confiabilidade do instrumento (Maroco \& Garcia-Marques, 2006). Logo, considerando os ótimos indicadores da PSS-4 na AFC, entende-se que o alfa reduzido possa estar relacionado à menor variabilidade produzida por apenas dois itens (Tavakol \& Dennick, 2011), o que também foi salientado por Leung et al. (2010) em discussão semelhante acerca da PSS-4.

Já com a PSS-10, detectou-se o que se poderia considerar como uma solução harmônica entre as versões completa e mais reduzida da PSS, pois ela agrega excelentes indicadores de qualidade na $\mathrm{AFC}$, visando ao intento de uma escala reduzida para o estresse. Esse achado já foi salientado por outros pesquisadores (Cohen \& Janicki-Deverts, 2012), os quais, embora não descartem a aplicação da PSS-14, apontam a PSS-10 como sendo a medida sucinta para o estresse, tendo em vista a quantidade de itens e indicadores de validade. A PSS-4, por sua vez, seria preferencial nas pesquisas de cunho epidemiológico e com objetivo de realizar um screen geral do estresse (Andreou et al., 2011; Herrero \& Meneses, 2006; Leung et al., 2010), isso por sua praticidade, ainda que produza menor variabilidade nas respostas.

Nas AFC's se constatou que o modelo unifatorial não apresentou ajuste satisfatório na PSS-14 e PSS10 e mostrou ajuste limítrofe no RMSEA da PSS-4, contraindicando a utilização de apenas um fator. Inclusive, o modelo bifatorial, além de exibir ajustes adequados nas versões com 14 e 10 itens, na PSS-4 alcançou parâmetros perfeitos nos índices GFI, CFI e 
RMSEA, o que, embora seja raro em uma amostra acima de 400 indivíduos, é explicado pelo fato do modelo ter somente um grau de liberdade e o valor do qui-quadrado impactar no cálculo dos índices utilizados (Byrne, 2010; Garson, 2012); portanto, isso não invalida a fiabilidade do achado. Os índices CAIC, que serve à comparação de modelos, e ECVI, que testa a capacidade de replicação em outras amostras equivalentes, somam-se às evidências que o modelo bifatorial é o mais adequado, pois corroborou o ajuste nas três versões, chegando a serem praticamente $50 \%$ inferiores ao unifatorial paralelo em todas as condições analisadas.

A consideração da escala PSS em dois fatores, além de manter o modelo original de Cohen et al. (1983), suscita a análise de que cada fator remete a um construto distinto na compreensão do fenômeno do estresse (Cerclé, Gadéa, Hartmann, \& Lourel, 2008; Leung et al., 2010). Com efeito, o fator negativo parece objetivamente relacionado à uma concepção dual do estresse, neste caso, representando o distress, que é o estado de sofrimento ou desgaste provocado ao sujeito se deparar com condições adversas e não possuir recursos capazes de lidar com a situação (Cohen et al., 2007; Faro \& Pereira, 2013; Goldstein \& Kopin, 2007). No positivo, refere-se à dimensão de avaliação no enfrentamento dos estressores, desenvolvida na teoria do coping (Lazarus \& Folkman, 1984), que se trata da capacidade percebida pelos próprios indivíduos de confrontar-se com estressores no seu cotidiano e, ainda assim, manter adequados níveis de bem-estar e saúde. Logo, entende-se que além do modelo bifatorial permitir a avaliação do estado estresse, ele pode favorecer ao escrutínio da relação "exposição (fator negativo) x proteção (fator positivo)", embora mais estudos devam ser conduzidos nessa perspectiva.

Nas cargas fatoriais, constatou-se que todas as escalas tiveram itens com carga satisfatória $(>0,400)$, exceto na PSS-14, em que o item 12 (Neste último mês, com que frequência você tem se encontrado pensando sobre as coisas que deve fazer?) ficou abaixo desse ponto de corte. Tal item é a tradução de Luft et al. (2007) para a pergunta: "In the last month, how often have you found yourself thinking about things that you have to accomplish?" da escala original. Em virtude da tradução, ainda que se tenha mantido a noção exata da frase, talvez a expressão "se encontrado pensando..." não tenha refletido necessariamente um estressor no contexto pesquisado, conforme era esperado.

Vale ressaltar que o baixo valor da carga fatorial do item 12 também foi detectado no estudo de Ramírez e Hernández (2007) com uma amostra mexicana, sendo que os autores propuseram, além da tradução, a adaptação do conteúdo ao contexto da cultural local. Seguindo essa ideia, acredita-se que a reformulação do item, ao inserir o sentido de "preocupação", possa tornar mais evidente a propriedade estressógena do enunciado, sendo uma sugestão: Neste último mês, com que frequência você tem se sentido preocupado, pensando sobre as coisas que deve fazer? Com essa modificação, que pode ser testada em futuros estudos, espera-se alcançar um significado mais congruente ao contexto brasileiro.

Embora tenham sido constatadas correlações significativas, porém fracas, na relação entre as versões da PSS e o QSG, os achados ratificaram a convergência entre as escalas, tal como visto em pesquisas similares (Chaaya, Osman, Naassan, \& Mahfoud, 2010; A. M. L. Lee et al., 2007; Örücü \& Demir, 2009). Vale salientar que ainda que seja uma escala amplamente utilizada para mensuração da saúde mental e bem-estar psicológico (Gouveia et al., 2012) e os autores das pesquisas aqui referidas a utilizem como baliza de análise da PSS, a QSG não é uma medida propriamente de estresse, apesar de existir autores que defendam o contrário (ver Gouveia et al., 2012). Noutras palavras, a QSG não reflete necessariamente um padrão-ouro de comparação da PSS e isso pode ajudar a explicar o índice de correlação obtido $(<0,30)$.

Diante do exposto, faz-se a ressalva de que são necessários mais estudos que averiguem a relação "QSG x PSS" no contexto brasileiro e, também, investigações que utilizem medidas fisiológicas de fácil coleta para análise da convergência da PSS, as quais possuem o status de padrão-ouro no estudo do estresse, a exemplo do cortisol (Cohen et al., 2007; Faro \& Pereira, 2013; Holsboer \& Ising, 2010). Enfim, a despeito da constatação da reciprocidade entre o estresse e a saúde geral observada neste estudo, entende-se que a escolha da QSG para análise da validade convergente se mostrou como uma limitação da presente pesquisa, requerendo cautela na aplicação desses achados.

Como previsto, além da representatividade populacional, a amostragem se aproximou da proporcionalidade entre os sexos, com maior número de mulheres, assim como no último Censo do Instituto Brasileiro de Geografia e Estatística (IBGE, 2010), que foi de aproximadamente $55 \%$ em Aracaju. Inclusive, as demais variáveis da caracterização também ficaram próximas ao levantado no Censo 2010, tais como o predomínio de pessoas entre 20 e 40 anos, ocupadas laborativamente, com a maioria tendo até o ensino médio e renda média em pouco mais de três salários mínimos, além de cerca de $10 \%$ de tabagistas.

No estresse, a média de pontuação foi de aproximadamente 24 pontos com a PSS-14, o que é exatamente o ponto médio da escala na presente distribuição. Utilizando-se da PSS-14, médias mais altas foram detectadas em pesquisas na Jordânia $(26,3 ; D P=8,20$; Almadi et al., 2012), Espanha (25,0; $D P=8,10$; Remor, 2006), Japão $(27,6$; $D P=8,42$; Mimura \& Grifftihs, 2008) e Grécia $(25,0$; $D P=7,90$; Katsarou et al., 2012). Por sua vez, o valor aqui encontrado foi bem próximo a um estudo nos EUA $(24,2 ; D P=7,93$; Lavoie \& Douglas, 2012) e mais baixo em comparação a pesquisas na China $(22,9 ; D P=5,85$; Leung et al., 2010), México (21,9; $D P=7,03$; Ramírez \& Hernández, 2007), Grécia $(21,4 ; D P=6,61$; Andreou et al., 2011) e à validação inicial realizada no Brasil $(21,4$; $D P=7,69$; Luft et al., 2007).

Com a PSS-10, a média desta amostra foi de 17 pontos, estando próxima à obtida na Espanha $(17,6 ; D P=6,70$; 
Faro, A. (2015). Análise Fatorial Confirmatória das Três Versões da Perceived Stress Scale (PSS): Um Estudo Populacional.

Remor, 2006). Entretanto, o valor médio aqui obtido foi maior que o observado na Grécia $(14,9 ; D P=5,29$; Andreou et al., 2011), na China em $2010(15,7 ; D P=4,60$; Leung et al., 2010) e em 2011 (15,2; $D P=5,60$; Wang et al., 2011), nos EUA $(15,8 ; D P=7,50$; Cohen $\&$ Janicki-Deverts, 2012) e em Portugal (15,0; $D P=6,40$; Trigo, Canudo, Branco, \& Silva, 2010; 15,5; $D P=6,11$; Ribeiro $\&$ Marques, 2009). Já na PSS-4, a média desta amostragem ficou em 6 pontos, basicamente igual à encontrada na China $(6,2 ; D P=2,05$; Leung et al., 2010) e menor que a vista na Catalunha $(9,8 ; D P=2,34$; Herrero \& Meneses, 2006). Vê-se que, de modo geral, a média populacional do estresse com a PSS-14 em Aracaju (SE) tendeu a ser menor, enquanto que na PSS-10 maior. No caso da PSS-4, que possui menos estudos, apenas o índice da Catalunha ficou mais distante. Com efeito, considerando que este é o primeiro estudo populacional com a PSS no Brasil, tais indicadores internacionais podem servir como parâmetro de avaliação do estresse no país.

Quando analisadas as pontuações médias da PSS por sexo, em suas três versões, o achado foi consistente com outros estudos (Cohen \& Janicki-Deverts, 2012; Kajantie, 2008; Lavoie \& Douglas, 2012; Wang et al., 2011), em que as mulheres tendem a exibir maior média e, em particular, no fator negativo das escalas. Quanto às demais variáveis sociodemográficas, a PSS-14 mostrou maior capacidade discriminativa, pois todas denotaram significância estatística na comparação entre estratos, o que concorda com outras investigações (faixa etária, Hur, MacGregor, Cherkas, Williams, \& Spector, 2012; escolaridade, faixa de renda e ocupação, Abbott, 2007; tabagismo, Leung et al., 2010). No caso da PSS-10, somente na faixa etária não houve diferenças significativas e, na PSS-4, não houve com a ocupação e, novamente, a faixa etária não variou significativamente. Dado o exposto, percebe-se que através da PSS é possível detectar diferenças em estratos populacionais quanto à prevalência do estresse, isto é, a escala possui capacidade de identificar grupos que podem estar mais expostos, ou mais protegidos, frente ao estresse.

Através da normatização com o escore T para as três versões da PSS, na amostra total e por sexo, forneceram-se parâmetros de comparação para futuras pesquisas, dispondo as pontuações obtidas segundo a distribuição populacional. Logo, os percentis 5 e 95 mostram os estratos extremos da proporcionalidade do estresse, ou seja, estabelecem-se possíveis pontos de corte para detecção de indivíduos que se situam nos clusters de maior e menor estresse. Somado a isso, o percentil 50 caracterizou o parâmetro médio de avaliação das versões da PSS, caso se deseje a formulação de um indicador categórico para avaliação do fenômeno. A exemplo dessa possibilidade, estratificando-se a pontuação em 5 níveis [percentis 25 (baixo), 50 (normal), 75 (moderado), 90 (alto) e 95 (muito alto)], teríamos, na população total, os seguintes intervalos da pontuação: $\leq 18$ (baixo), 19-24 (normal), 25-29 (moderado), 30-35 (alto) e $>35$ (muito alto). O mesmo poderia ser feito por sexo.
Como limitações desta pesquisa, vale a pena ressaltar que os resultados obtidos, embora objetivando a representatividade populacional, não se aplica à compreensão do estresse em dois importantes grupos: as crianças e os idosos acima de 70 anos. Ademais, também não é adequado fazer inferências a respeito do estresse em portadores de enfermidades agudas ou crônicas, ou ainda, desordens físicas ou mentais incapacitantes, tendo em vista os critérios amostrais então delineados. Portanto, uma sugestão para novas pesquisas é o levantamento e normatização, em escala populacional, dos valores nesses grupos, considerando-se as peculiaridades da ocorrência do estresse na infância, terceira idade e condições de adoecimento e/ ou limitação funcional.

Ainda como proposta para novas investigações, a verificação da validade da estratificação dos níveis do estresse (e.g., análises de Curvas ROC com escalas diagnósticas de transtornos de humor), a condução de pesquisas populacionais (transversais ou longitudinais) em outras cidades ou regiões do Brasil, cujos objetivos possam também ser a normatização dos escores e a análise das propriedades psicométricas, permitiria o maior conhecimento quanto à distribuição social do estresse e suas peculiaridades, teóricas e empíricas, na realidade nacional.

\section{Referências}

Abbott, S. (2007). The psychosocial effects on health of socioeconomic inequalities. Critical Public Health, 17, 151-158. doi:10.1080/09581590701225862

Almadi, T., Cathers, I., Mansour, A. M. H., \& Chow, C. M. (2012). An Arabic version of the Perceived Stress Scale: Translation and validation study. International Journal of Nursing Studies, 49, 84-89. doi:10.1016/j.ijnurstu.2011.07.012

Andreou, E., Alexopoulos, E. C., Lionis, C., Varvogli, L., Gnardellis, C., Chrousos, G. P., \& Darviri, C. (2011). Perceived Stress Scale: Reliability and validity study in Greece. International Journal of Environmental Research and Public Health, 8, 3287-3298. doi:10.3390/ijerph8083287

Byrne, B. (2010). Structural equation modeling with AMOS. Basic concepts, applications, and programming ( $2^{\text {nd }}$ ed.). New York: Taylor \& Francis Group.

Instituto Brasileiro de Geografia e Estatística. (2010). Sinopse do censo demográfico de Sergipe [Arquivo de dados]. Recuperado em http://www.ibge.gov.br/estadosat/imprimir. php? sigla $=$ se\&tema $=$ sinopse_censodemog 2010

Cerclé, A., Gadéa, C., Hartmann, A., \& Lourel, M. (2008). Typological and factor analysis of the perceived stress measure by using the PSS scale. Revue Européenne de Psychologie Appliquée, 58, 227-239. doi:10.1016/j.erap.2008.09.006

Chaaya, M., Osman, H., Naassan, G., \& Mahfoud, Z. (2010). Validation of the Arabic version of the Cohen Perceived Stress Scale (PSS-10) among pregnant and postpartum women. BMC Psychiatry, 10, 111-118. doi:10.1186/1471-244X-10-111

Cohen, S., \& Janicki-Deverts, D. (2012). Who's stressed? Distributions of psychological stress in the United States in probability samples from 1983, 2006, and 2009. Journal of Applied Social Psychology, 42, 1320-1334. doi:10.1111/j.15591816.2012.00900.x 
Cohen, S., Janick-Deverts, D., \& Miller, G.E. (2007). Psychological stress and disease. JAMA, 298, 1685-1687. doi:10.1001/ jama.298.14.1685

Cohen, S., Kamarck, T., \& Mermelstein, R. (1983). A global measure of perceived stress. Journal of Health and Social Behavior, 24, 385-396.

Faro, A. (2013). Estresse e estressores na pós-graduação: Estudo com mestrandos e doutorandos no Brasil. Psicologia: Teoria e Pesquisa, 29, 51-60. doi:10.1590/S0102-37722013000100007

Faro, A., \& Pereira, M. E. (2013). Estresse: Revisão narrativa da evolução conceitual, perspetivas teóricas e metodológicas. Psicologia, Saúde \& Doenças, 14, 78-100.

Garson, D. G. (2012). Structural equation modeling. Asheboro, NC: Statistical Association.

Goldstein, D. S., \& Kopin, I. J. (2007). Evolution of concepts of stress. Stress, 10, 10-120.

Gouveia, V. V., Chaves, S. S. da S., Oliveira, I. C. P., Dias, M. R., Gouveia, R. S. V., \& Andrade, P. R. (2003). A utilização do QSG-12 na população geral: Estudo de sua validação de construto. Psicologia: Teoria e Pesquisa, 19(3), 241-248.

Gouveia, V. V., Lima, T. J. S., Gouveia, R. S. V., Freires, L. A., \& Barbosa, L. H. G. M. (2012). Questionário de Saúde Geral (QSG-12): O efeito de itens negativos em sua estrutura fatorial. Caderno de Saúde Pública, 28, 375-384. doi:10.1590/ S0102-311X2012000200016

Herrero, J., \& Meneses, J. (2006). Short web-based versions of the perceived stress (PSS) and Center for Epidemiological Studies-Depression (CESD) scales: A comparison to pencil and paper responses among internet users. Computers in $\mathrm{Hu}$ man Behavior, 22, 830-846. doi:10.1016/j.chb.2004.03.007

Holsboer, F., \& Ising, M. (2010). Stress hormone regulation: Biological role and translation into therapy. Annual Review of Psychology, 61, 81-109. doi:10.1146/annurev. psych.093008.100321

Hur, Y.-M., MacGregor, A. J., Cherkas, L., Williams, F. M. K., \& Spector, T. D. (2012). Age differences in genetic and environmental variations in stress-coping during adulthood: A study of female twins. Behavior Genetics, 42, 541-548. doi:10.1007/s10519-012-9541-2

Kajantie, E. (2008). Physiological stress response, estrogen, and the male-female mortality gap. Current Directions in Psychological Science, 17, 348-352. doi:10.1111/j.14678721.2008.00604.x

Katsarou, A., Panagiotakos, D., Zafeiropoulou, A., Vryonis, M., Skoularigis, I., Tryposkiadis, F., \& Papageorgiou, C. (2012). Validation of a Greek version of PSS-12: A global measure of perceived stress. Central European Journal of Public Health, 20, 104-109.

Lavoie, J. A. A., \& Douglas, K. S. (2012). The Perceived Stress Scale: Evaluating configural, metric and scalar invariance across mental health status and gender. Journal of Psychopathology and Behavioral Assessment, 34, 48-57. doi:10.1007/ s10862-011-9266-1

Lazarus, R. S., \& Folkman, S. (1984). Stress, appraisal and coping. New York: Springer.

Lee, E. H. (2012). Review of the psychometric evidence of the Perceived Stress Scale. Asian Nursing Research, 6, 121-127. doi:10.1016/j.anr.2012.08.004

Lee, A. M. L., Wong, J. G. W. S., McAlonan, G. M., Cheung, V., Cheung, C., Sham, P. C., ...Chua, S. E. (2007). Stress and psychological distress among SARS survivors 1 year after the outbreak. The Canadian Journal of Psychiatry, 52, 233-240.
Leung, D. Y. P., Lam, T. H., \& Chan, S. S. C. (2010). Three versions of Perceived Stress Scale: Validation in a sample of Chinese cardiac patients who smoke. BMC Public Health, 10, 513-520. doi:10.1186/1471-2458-10-513

Luft, C. D. B., Sanches, S. O., Mazo, G. Z., \& Andrade, A. (2007). Versão brasileira da Escala de Estresse Percebido: Tradução e validação para idosos. Revista de Saúde Pública, 41, 606-615. doi:10.1590/S0034-89102007000400015

Maroco, J., \& Garcia-Marques, T. (2006). Qual a fiabilidade do alfa de Cronbach? Questões antigas e soluções modernas. Laboratório de Psicologia, 4, 65-90.

McEwen, B. S. (2008). Central effects of stress hormones in health and disease: Understanding the protective and damaging effects of stress and stress mediators. European Journal of Pharmacology, 583, 174-185. doi:10.1016/j. ejphar.2007.11.071

Mimura, C., \& Griffiths, P. (2008). A Japanese version of the Perceived Stress Scale: Cross-cultural translation and equivalence assessment. BMC Psychiatry, 8, 1-7. doi:10.1186/1471244X-8-85

Monroe, S. M. (2008). Modern approaches to conceptualizing and measuring human life stress. Annual Review of Clinical Psychology, 4, 33-52. doi:10.1146/annurev. clinpsy.4.022007.141207

Örücü, M. C., \& Demir, A. (2009). Psychometric evaluation of Perceived Stress Scale for Turkish university students. Stress and Health, 25, 103-109. doi:10.1002/smi.1218

Ramírez, M. T. G., \& Hernández, R. L. (2007). Factor structure of the Perceived Stress Scale (PSS) in a sample from Mexico. The Spanish Journal of Psychology, 10, 199-206.

Reis, R. S. R., Hino, A. A. F., \& Añez, C. R. R. (2010). Perceived Stress Scale: Reability and validity study in Brazil. Journal of Health Psychology, 15, 107-114. doi:10.1177/1359105309346343.

Remor, E. (2006). Psychometric properties of a European Spanish version of the Perceived Stress Scale (PSS). The Spanish Journal of Psychology, 9, 86-93.

Ribeiro, J. P., \& Marques, T. (2009). A avaliação do stresse: A propósito de um estudo de adaptação da Escala de Percepção de Stresse. Psicologia, Saúde \& Doenças, 10, 237-248.

Tabachnick, B. G., \& Fidel, L. S. (2007). Using multivariate statistics $\left(5^{\text {th }}\right.$ ed.). New York: Harper Collins.

Tavakol, M., \& Dennick, R. (2011). Making sense of Cronbach's alpha. International Journal of Medical Education, 2, 53-55. doi:10.5116/ijme.4dfb.8dfd

Trigo, M., Canudo, N., Branco, F., \& Silva, D. (2010). Estudo das propriedades psicométricas da Perceived Stress Scale (PSS) na população portuguesa. Psychologica, 53, 353-378.

Wang, Z., Chen, J., Boyd, J. E., Zhang, H., Jia, X., Qiu, J., \& Xiao, Z. (2011). Psychometric properties of the Chinese version of the Perceived Stress Scale in police women. Plos One, 6(12): e28610. doi:10.1371/journal.pone.0028610 